\title{
65 Aniversario del Boletín de la Sociedad BotánICA de MéxICO (1944 - 2009)
}

C on este segundo número de 2009 del Boletín de la Sociedad Botánica de México se cumplen 65 años desde el lanzamiento inicial de esta revista científica, órgano oficial de la Sociedad Botánica de nuestro país, que permite llevar a la luz pública el trabajo científico de investigadores mexicanos y extranjeros relacionado principalmente con las plantas mexicanas y las poblaciones y comunidades vegetales que éstas conforman.

A algunas personas podría parecer que 65 años para una revista científica no es mucho tiempo, pero en realidad sí lo es. Para comenzar, podemos decir que esta edad da un lugar al Boletín en el selecto grupo de las revistas científicas más longevas del continente americano y posiblemente del mundo entero. Pero también podemos pensar en otros argumentos más alejados del mundo de la ciencia que ilustren esta afirmación. Por ejemplo, 65 años es el promedio mundial de esperanza de vida de un individuo nacido en este año, según la CIA (Agencia Central de Inteligencia de los Estados Unidos; https://www.cia.gov/library/publications/the-worldfactbook/rankorder/2102rank.html) y rebasa el tiempo que ha existido la República Popular China, país que este año cumplió 60 años, periodo en el que pasó de ser un estado comunista a una de las potencias capitalistas más poderosas del orbe. Asimismo, 65 años es prácticamente el tiempo transcurrido desde que concluyó la segunda gran guerra mundial del siglo XX, la cual marcó parte del mundo de manera imborrable. En ese lapso emergió y cayó la Cortina de Hierro, se encendió y extinguió la Guerra Fría, los seres humanos se pasearon por el espacio y pusieron pie en la luna por vez primera, proliferaron enfermedades antes desconocidas, surgieron nuevas naciones y se desvanecieron otras, se erigieron y cayeron terribles dictaduras y se colapsaron edificios icónicos. La población mundial cambió de un poco más de dos mil millones de personas en 1944 a cerca de 7 mil millones en 2009. Otras cosas, lamentablemente, han seguido igual: prevalece la desigualdad social, sigue habiendo pocos países ricos y muchos otros muy pobres, los conflictos de todo tipo a nivel mundial se prenden y apagan constantemente, de manera semejante a los foquitos de las series navideñas.
Los últimos 65 años han atestiguado la época más terrible del deterioro del ambiente en México, y a pesar de esfuerzos heroicos por parte de numerosos individuos y organizaciones encaminados a evitar este proceso, parece cada vez más que el deterioro ambiental no conmueve demasiado a la enorme mayoría de la población humana. Por ello, es muy importante destacar que durante este periodo también han sido notables los avances de la investigación botánica en México. Así lo confirman los recuentos que se han hecho de los artículos publicados en el Boletín desde su nacimiento (Butanda-Cervera, 1981; Magaña-Rueda, 1997; RomeroRomero y Meave, 2007) y el surgimiento y crecimiento de otras publicaciones especializadas en el área, en particular de nuestra revista hermana Acta Botanica Mexicana, e inclusive el crecimiento vertiginoso del número de artículos publicados en revistas extranjeras que versan sobre este tópico. De ser una actividad que hace 65 años se practicaba casi exclusivamente en la ciudad de México, actualmente la botánica se cultiva en instituciones educativas y de investigación en todo el territorio nacional.

El poder dar continuidad a la publicación del Boletín no ha sido siempre fácil. En realidad, sólo en los últimos 16 años ha sido posible mantener un ritmo de publicación de dos números al año de forma ininterrumpida, si bien no siempre con suficiente puntualidad, a pesar de que en 11 años anteriores no consecutivos $(1945,1948,1949,1950$, $1951,1954,1957,1958,1975,1982$ y 1983) ya se había alcanzado esta tasa anual de aparición. Además, no podemos pasar por alto el hecho de que en nueve años (12.3\% del total) de este periodo (1964, 1966, 1967, 1968, 1971, 1973, 1978, 1985 y 1986) no salió a la luz ningún fascículo de la revista.

El cuadro 1 presenta una lista de las personas que han tenido en sus manos la responsabilidad de esta empresa. Esta lista se construyó a partir de información extraída de los propios ejemplares de la revista. Sin duda, el mayor esfuerzo lo han hecho los editores o los miembros del comité editorial, junto con los editores ejecutivos y figuras semejantes que han contribuido de diversas formas hacia este fin común. Sin embargo, el trabajo de todas estas personas quizá no habría sido posible o habría enfrentado obstáculos 
aun mayores sin el apoyo decidido, la confianza y la indispensable libertad de acción otorgados por los numerosos presidentes de la Sociedad Botánica de México.

En esta lista guarda un primerísimo lugar, tanto por razón de cronología como por el gran número de años que dedicó a la edición del Boletín, el Profesor Maximino Martínez, quien se encargó de manera única o colegiada de la aparición de los 28 números de la revista correspondientes a sus primeros 20 años de existencia. Sirva la compilación de todos estos nombres como un humilde homenaje y un recordatorio a los que ahora practican la botánica, sobre todo los más jóvenes, de que detrás de esta cada vez más larga trayectoria han estado hombres y mujeres entregados a este proyecto académico con una convicción que con frecuencia tiene tintes de pasión. Esta información también nos ayuda a visualizar la importancia de que todos los miembros de la Sociedad Botánica de México y de muchas personas más dedicadas al trabajo botánico contribuyan de manera efectiva y directa a la elaboración de la revista, por ejemplo publicando en ella algunos de nuestros mejores trabajos (llamado que ya habían hecho Lot [1994] y Lot y Butanda [1994], ¡hace 15 años!), y que no dejemos descansar la responsabilidad de su existencia en una o unas pocas personas. Baste señalar que el primer hueco en la trayectoria de continuidad del Boletín corresponde precisamente con el año de las muertes del Prof. Martínez y del Dr. Miranda. La lección es clara y todos deberíamos asumir el compromiso con la mayor energía.

Es momento de celebrar un sexagésimo quinto aniversario en medio de un entorno social, económico y ambiental difícil. Quizá el poder ver hacia atrás por un momento y ponderar el camino recorrido sirva de inspiración y nos recuerde que es posible mantener vivo un ideal y un proyecto científico nacional, inclusive en circunstancias verdaderamente adversas.

\section{Agradecimientos}

Agradezco la ayuda de Marco A. Romero durante la compilación y verificación de la información utilizada en esta nota. Antonio Lot proporcionó y corroboró información histórica valiosa y me hizo notar errores en una versión previa, sobre la que también Alberto Gallardo, Eduardo A. Pérez García, Hugo Tovar y Marco Antonio Romero hicieron comentarios muy útiles.

\section{Literatura citada}

Butanda-Cervera, A. 1981. Índice acumulativo del Boletín de la Sociedad Botánica de México de los números 1 (1944) al 40 (1981). Boletín de la Sociedad Botánica de México 40:73-132.

Lot A. 1994. Destino de nuestras contribuciones botánicas: el caso de las revistas periódicas mexicanas. Macpalxóchitl 133:3-9.

Lot A. y Butanda A. 1994. El Boletín de la Sociedad Botánica de México en el contexto de las publicaciones científicas. Boletín de la Sociedad Botánica de México 55:59-64.

Lot A. y Carvajal M. 1981. Cronología de los principales acontecimientos históricos de la Sociedad Botánica de México. Boletín de la Sociedad Botánica de México 40:25-43.

Magaña-Rueda, P. 1997. Índice acumulativo del Boletín de la Sociedad Botánica de México del número 41 (1981) al número 60 (1997). Boletín de la Sociedad Botánica de México 61:105-137.

Romero-Romero M.A. y J.A. Meave. 2007. Lista de contribuciones publicadas en el Boletín de la Sociedad Botánica de México durante el periodo enero de 1944 a junio de 2007. Boletín de la Sociedad Botánica de México 80:113-138.

\section{Jorge A. Meave \\ Facultad de Ciencias \\ Universidad Nacional Autónoma de México \\ jorge.meave@ciencias.unam.mx}

Cuadro 1. Recuento cronológico de la publicación de todos los números del Boletín de la Sociedad Botánica de México aparecidos hasta la fecha. Se indica el nombre del presidente en turno de la Sociedad Botánica de México, así como del editor o de los miembros del comité editorial de la revista.

\begin{tabular}{ccll}
\hline Año & No. & Presidente en turno & Editor(es) del Bol. Soc. Bot. Méx. \\
\hline \multirow{2}{*}{1944} & $1^{1}$ & Maximino Martínez & Maximino Martínez \\
1945 & 2 & Maximino Martínez & Maximino Martínez \\
& 3 & Ángel Roldán & Maximino Martínez \\
\hline 1946 & 4 & Ángel Roldán & Maximino Martínez \\
\hline 1947 & 5 & Ángel Roldán & Maximino Martínez \\
1948 & 6 & Ángel Roldán/Gabriel Itié & Maximino Martínez \\
& 7 & Gustavo Aguirre Benavides & Maximino Martínez \\
\hline 1949 & $8^{2}$ & Gustavo Aguirre Benavides & Maximino Martínez \\
& 9 & Gustavo Aguirre Benavides & Maximino Martínez \\
\hline 1950 & 10 & Ladislao Paray & Maximino Martínez \\
& 11 & Efraím Hernández Xolocotzi & Maximino Martínez \\
\hline 1951 & 12 & Efraím Hernández Xolocotzi & Maximino Martínez \\
& 13 & Glenn Davis & Maximino Martínez \\
\hline
\end{tabular}




\begin{tabular}{|c|c|c|c|}
\hline Año & No. & Presidente en turno & Editor(es) del Bol. Soc. Bot. Méx. \\
\hline 1952 & 14 & Glenn Davis & Maximino Martínez \\
\hline 1953 & 15 & Ladislao Paray & Maximino Martínez \\
\hline \multirow[t]{2}{*}{1954} & 16 & Efraím Hernández Xolocotzi & Maximino Martínez \\
\hline & 17 & Efraím Hernández Xolocotzi & Maximino Martínez \\
\hline 1955 & 18 & Efraím Hernández Xolocotzi & Maximino Martínez \\
\hline 1956 & 19 & Efraím Hernández Xolocotzi & Maximino Martínez \\
\hline \multirow[t]{2}{*}{1957} & 20 & Efraím Hernández Xolocotzi & Maximino Martínez \\
\hline & 21 & Efraím Hernández Xolocotzi & Maximino Martínez \\
\hline \multirow[t]{2}{*}{1958} & 22 & Alberto J. Flores & Maximino Martínez \\
\hline & 23 & Alberto J. Flores & Maximino Martínez \\
\hline 1959 & 24 & Alberto J. Flores & Maximino Martínez \\
\hline 1960 & 25 & $\begin{array}{l}\text { Jerzy Rzedowski } \\
\text { Faustino Miranda }\end{array}$ & Maximino Martínez, Gastón Guzmán, Efraím Hernández X., \\
\hline 1961 & 26 & $\begin{array}{l}\text { Jerzy Rzedowski } \\
\text { Faustino Miranda }\end{array}$ & Maximino Martínez, Gastón Guzmán, Efraím Hernández X., \\
\hline 1962 & 27 & Arturo Gómez-Pompa & $\begin{array}{l}\text { Maximino Martínez, Faustino Miranda, Efraím Hernández X., } \\
\text { Jesús Vázquez Soto }\end{array}$ \\
\hline 1963 & 28 & Arturo Gómez-Pompa & $\begin{array}{l}\text { Efraím Hernández X., Faustino Miranda, Maximino Martínez, } \\
\text { Ramón Riba }\end{array}$ \\
\hline 1964 & & Efraím Hernández Xolocotzi ${ }^{3}$ & \\
\hline 1965 & 29 & Efraím Hernández Xolocotzi & Efraím Hernández X. y Ramón Riba \\
\hline 1966 & & Javier Valdés ${ }^{3}$ & \\
\hline 1967 & & Javier Valdés ${ }^{3}$ & \\
\hline 1968 & & Javier Valdés ${ }^{3}$ & \\
\hline 1969 & 30 & Ramón Echenique-Manrique & $\begin{array}{l}\text { Jerzy Rzedowski, Arturo Gómez-Pompa, Efraím Hernández X., } \\
\text { Ramón Riba }\end{array}$ \\
\hline 1970 & 31 & Ramón Echenique-Manrique & $\begin{array}{l}\text { Jerzy Rzedowski, Arturo Gómez-Pompa, Efraím Hernández X., } \\
\text { Roberto Cruz Cisneros }\end{array}$ \\
\hline 1971 & & Ramón Echenique-Manrique ${ }^{3}$ & \\
\hline 1972 & 32 & José Sarukhán K. & $\begin{array}{l}\text { Jerzy Rzedowski, Arturo Gómez Pompa, Efraím Hernández X., } \\
\text { Roberto Cruz Cisneros }\end{array}$ \\
\hline 1973 & & José Sarukhán K. ${ }^{3}$ & \\
\hline 1974 & $33^{4}$ & José Sarukhán K. & $\begin{array}{l}\text { Mario Sousa Sánchez* (J. Rzedowski, E. Hernández X., } \\
\text { C. Delgadillo M.) }\end{array}$ \\
\hline \multirow[t]{2}{*}{1975} & 34 & José Sarukhán K. & $\begin{array}{l}\text { Mario Sousa Sánchez* (J. Rzedowski, E. Hernández X., } \\
\text { C. Delgadillo M.) }\end{array}$ \\
\hline & 35 & José Sarukhán K. & $\begin{array}{l}\text { Mario Sousa Sánchez* (J. Rzedowski, E. Hernández X., } \\
\text { C. Delgadillo M.) }\end{array}$ \\
\hline 1976 & 36 & Magdalena Peña de Sousa & $\begin{array}{l}\text { Mario Sousa Sánchez* (J. Rzedowski, J. Sarukhán, } \\
\text { A. Butanda C., G. Guzmán, E. Hernández X. y E.M. Engleman) }\end{array}$ \\
\hline 1977 & 37 & Magdalena Peña de Sousa & $\begin{array}{l}\text { Mario Sousa Sánchez* (J. Rzedowski, J. Sarukhán, } \\
\text { A. Butanda C., G. Guzmán, E. Hernández X. y E.M. Engleman) }\end{array}$ \\
\hline 1978 & & Francisco González Medrano ${ }^{3}$ & \\
\hline 1979 & 38 & Francisco González Medrano & $\begin{array}{l}\text { Mario Sousa Sánchez* (J. Rzedowski, M.M. Engleman, E. } \\
\text { Hernández X., J. Sarukhán K., A. Chimal Hernández, V. Toledo M.) }\end{array}$ \\
\hline 1980 & 39 & Antonio Lot Helgueras & $\begin{array}{l}\text { Carlos Vázquez Yanes* (J. Rzedowski, E. Mark Engleman. } \\
\text { E. Hernández X., J. Sarukhán K., V. Sosa, V. Toledo M.) }\end{array}$ \\
\hline 1981 & 40 & Antonio Lot Helgueras & $\begin{array}{l}\text { Carlos Vázquez Yanes* (J. Rzedowski, E.M. Engleman. } \\
\text { E. Hernández X., J. Sarukhán K. V. Sosa, V. Toledo M.) }\end{array}$ \\
\hline & 41 & Antonio Lot Helgueras & $\begin{array}{l}\text { Carlos Vázquez Yanes* (J. Rzedowski, E.M. Engleman. } \\
\text { E. Hernández X., J. Sarukhán, V. Sosa, V. Toledo M.) }\end{array}$ \\
\hline
\end{tabular}




\begin{tabular}{|c|c|c|c|}
\hline Año & No. & Presidente en turno & Editor(es) del Bol. Soc. Bot. Méx. \\
\hline \multirow[t]{2}{*}{1982} & 42 & Ramón Riba y Nava Esparza & $\begin{array}{l}\text { Carlos Vázquez Yanes* (J. Rzedowski, E.M. Engleman, } \\
\text { E. Hernández X., J. Sarukhán, R. Grether., V. Toledo) }\end{array}$ \\
\hline & 43 & Ramón Riba y Nava Esparza & $\begin{array}{l}\text { Carlos Vázquez Yanes* (J. Rzedowski, E.M. Engleman, } \\
\text { E. Hernández X., J. Sarukhán, R. Grether G., V. Toledo) }\end{array}$ \\
\hline \multirow[t]{2}{*}{1983} & 44 & Ramón Riba y Nava Esparza & $\begin{array}{l}\text { Carlos Vázquez Yanes* (J. Rzedowski, E.M. Engleman, } \\
\text { E. Hernández X., J. Sarukhán, R. Grether, V. Toledo) }\end{array}$ \\
\hline & $45^{5}$ & Ramón Riba y Nava Esparza & $\begin{array}{l}\text { Carlos Vázquez Yanes* (J. Rzedowski, E.M. Engleman, } \\
\text { E. Hernández X., J. Sarukhán, R. Grether, V. Toledo) }\end{array}$ \\
\hline 1984 & $46^{6}$ & Hernando Sánchez-Mejorada Rodríguez & $\begin{array}{l}\text { Antonio Lot Helgueras* (R. Dirzo, E.M. Engleman, R. Grether, } \\
\text { E. Hernández X., H. Quero Rico, A. Rodríguez Isassi); } \\
\text { editor asistente: Armando Butanda }\end{array}$ \\
\hline 1985 & & Hernando Sánchez-Mejorada ${ }^{3}$ & \\
\hline 1986 & & Hernando Sánchez-Mejorada ${ }^{3}$ & \\
\hline 1987 & 47 & Hernando Sánchez-Mejorada Rodríguez & $\begin{array}{l}\text { Antonio Lot Helgueras* (R. Dirzo, E.M. Engleman, R. Grether, } \\
\text { E. Hernández X., H. Quero Rico, A. Rodríguez Isassi); } \\
\text { editor asistente: Armando Butanda }\end{array}$ \\
\hline 1988 & $48^{7}$ & Sergio Guevara Sada & $\begin{array}{l}\text { Antonio Lot Helgueras* (R. Dirzo, E.M. Engleman, R. Grether, } \\
\text { E. Hernández X., H. Quero Rico, A. Mendoza Ochoa); } \\
\text { editor asistente: Armando Butanda }\end{array}$ \\
\hline 1989 & 49 & Sergio Guevara Sada & $\begin{array}{l}\text { Antonio Lot Helgueras* (R. Dirzo, E.M. Engleman, R. Grether, } \\
\text { E. Hernández X., H. Quero Rico, A. Mendoza Ochoa); } \\
\text { editor asistente: Armando Butanda }\end{array}$ \\
\hline 1990 & 50 & Sergio Guevara Sada & $\begin{array}{l}\text { Antonio Lot Helgueras* (R. Dirzo, E.M. Engleman, R. Grether, } \\
\text { E. Hernández X., H. Quero Rico, A. Mendoza Ochoa); } \\
\text { editor asistente: Armando Butanda }\end{array}$ \\
\hline 1991 & 51 & Francisco Javier Álvarez Sánchez & $\begin{array}{l}\text { Antonio Lot Helgueras* (R. Dirzo, E.M. Engleman, R. Grether, } \\
\text { H. Quero Rico, G. Zamudio Varela); editor asistente: } \\
\text { Armando Butanda }\end{array}$ \\
\hline 1992 & 52 & Francisco Javier Álvarez Sánchez & $\begin{array}{l}\text { Antonio Lot Helgueras* (R. Dirzo, E.M. Engleman, R. Grether, } \\
\text { A. Argueta, H. Quero Rico, G. Zamudio Varela). } \\
\text { Editor: Armando Butanda }\end{array}$ \\
\hline 1993 & 53 & Francisco Javier Álvarez Sánchez & $\begin{array}{l}\text { Antonio Lot Helgueras* (R. Dirzo, E.M. Engleman, R. Grether, } \\
\text { A. Argueta, H. Quero Rico, G. Zamudio Varela). } \\
\text { Editor: Armando Butanda }\end{array}$ \\
\hline 1994 & $55^{8}$ & Daniel Piñero Dalmau & $\begin{array}{l}\text { Antonio Lot Helgueras* (R. Dirzo, E.M. Engleman, R. Grether, } \\
\text { A. Argueta, H. Quero Rico, G. Zamudio Varela). } \\
\text { Editor: Armando Butanda; responsable de la edición: Ken Oyama } \\
\text { Ken Oyama }\end{array}$ \\
\hline 1995 & $\begin{array}{l}56 \\
57\end{array}$ & $\begin{array}{l}\text { Daniel Piñero Dalmau } \\
\text { Daniel Piñero Dalmau }\end{array}$ & $\begin{array}{l}\text { Ken Oyama } \\
\text { Ken Oyama }\end{array}$ \\
\hline 1996 & 58 & Daniel Piñero Dalmau & Ken Oyama, Patricia Magaña \\
\hline 1997 & $\begin{array}{l}60 \\
61\end{array}$ & $\begin{array}{l}\text { Daniel Piñero Dalmau } \\
\text { Ken Oyama }\end{array}$ & $\begin{array}{l}\text { Ken Oyama, Patricia Magaña } \\
\text { Eberto Novelo, Patricia Magaña }\end{array}$ \\
\hline 1998 & $\begin{array}{l}62 \\
63\end{array}$ & $\begin{array}{l}\text { Ken Oyama } \\
\text { Ken Oyama }\end{array}$ & $\begin{array}{l}\text { Eberto Novelo, Patricia Magaña } \\
\text { Eberto Novelo, Patricia Magaña }\end{array}$ \\
\hline 1999 & $\begin{array}{l}64 \\
65\end{array}$ & $\begin{array}{l}\text { Ken Oyama } \\
\text { Patricia Dávila Aranda }\end{array}$ & $\begin{array}{l}\text { Eberto Novelo, Patricia Magaña } \\
\text { Eberto Novelo, Patricia Magaña }\end{array}$ \\
\hline 2000 & $\begin{array}{l}66 \\
67\end{array}$ & $\begin{array}{l}\text { Patricia Dávila Aranda } \\
\text { Patricia Dávila Aranda }\end{array}$ & $\begin{array}{l}\text { Rafael Lira, Patricia Magaña } \\
\text { Rafael Lira, Patricia Magaña }\end{array}$ \\
\hline 2001 & 68 & Patricia Dávila Aranda & Rafael Lira, Patricia Magaña \\
\hline
\end{tabular}




\begin{tabular}{ccll}
\hline Año & No. & Presidente en turno & Editor(es) del Bol. Soc. Bot. Méx. \\
\hline \multirow{2}{*}{2002} & 69 & Patricia Dávila Aranda & Rafael Lira, Patricia Magaña \\
& 70 & Victoria Sosa Ortega & Jorge A. Meave \\
& 71 & Victoria Sosa Ortega & Jorge A. Meave \\
\hline 2003 & 72 & Victoria Sosa Ortega & Jorge A. Meave \\
& 73 & Victoria Sosa Ortega & Jorge A. Meave \\
\hline 2004 & 74 & Victoria Sosa Ortega & Jorge A. Meave \\
& 75 & Victoria Sosa Ortega & Jorge A. Meave \\
\hline 2005 & 76 & Miguel Martínez Ramos & Jorge A. Meave \\
& 77 & Miguel Martínez Ramos & Jorge A. Meave \\
& 78 & Miguel Martínez Ramos & Jorge A. Meave \\
& 79 & Miguel Martínez Ramos & Jorge A. Meave \\
& 80 & Miguel Martínez Ramos & Jorge A. Meave \\
& $80($ S) & Miguel Martínez Ramos & Jorge A. Meave con Javier Álvarez-Sánchez y Julia Carabias \\
& 81 & Miguel Martínez Ramos & Jorge A. Meave \\
\hline 2008 & 82 & Adolfo Espejo Serna & Victoria Sosa Ortega \\
& 83 & Adolfo Espejo Serna & Victoria Sosa Ortega \\
& 84 & Adolfo Espejo Serna & Victoria Sosa Ortega \\
& 85 & Adolfo Espejo Serna & Victoria Sosa Ortega \\
\hline
\end{tabular}

\section{Notas:}

${ }^{1}$ La información contenida en este cuadro fue extraída de la segunda de forros de los ejemplares del Boletín de la Sociedad Botánica de México. Cabe aclarar que en sus primeros años no se reconocía expresamente en la publicación la figura de editor.

2 En este número de solicita de forma explícita por primera vez que los manuscritos sean enviados a Maximino Martínez, secretario de la mesa directiva de la sociedad.

${ }^{3}$ Información obtenida de Lot y Carvajal (1981) o inferida a partir de la información precedente y posterior.

${ }^{4}$ Desde este momento y durante varios años la publicación de la revista estuvo bajo la responsabilidad de un comité de editores; el asterisco ${ }^{*}$ ) denota al Jefe del Comité Editorial y entre paréntesis se nombran los miembros de dicho comité, cuya participación aparentemente fue muy heterogénea.

${ }^{5}$ Número publicado en 1984; ${ }^{6}$ publicado en $1986 ;{ }^{7}$ publicado en 1989 (Lot y Butanda, 1994).

${ }^{8}$ A partir del número 55 (1994), el Comité Editorial adquirió un definición más convencional en el contexto de las publicaciones científicas internacionales y sus miembros dejaron de participar en la elaboración cotidiana de la revista; ahora su papel consiste más bien en vigilar la conducción académica y las políticas editoriales. 